\title{
Automated Building Information Modeling System for Building Interior to Improve Productivity of BIM-based Quantity Take-Off
}

\author{
Seong-Ah Kim ${ }^{1}$, Sangyoon Chin ${ }^{2}$, Su-Won Yoon ${ }^{3}$, Tae-Hong Shin ${ }^{4}$, Yea-Sang Kim ${ }^{5}$ and Cheolho Choi ${ }^{6}$ \\ ${ }^{1}$ Dept. of Civil, Architectural, and Environmental System Engineering, Sungkyunkwan Univ, Suwon 400- \\ 746, S. Korea; PH (82)31-290-7578; FAX (82)31-290-7570 ; e-mail : Kody25@skku.edu \\ 2Dept. of Civil, Architectural, and Environmental System Engineering, Sungkyunkwan Univ, Suwon 400- \\ 746, S. Korea ; PH (82)31- 290-7578; FAX (82)31-290-7570 ; e-mail : schin@skku.edu (Corresponding \\ Author) \\ ${ }^{3}$ Doalltech Co., Ltd, DMC Hi-Tech Industry Center 1580, Sangam-Dong, Mapo-Gu, Seoul, Korea 121-835 ; \\ PH (82)2- 555-9779; FAX (82) 2-5555-886 ; e-mail : yoonsuwon@doalltech.com \\ 4Dept. of Civil, Architectural, and Environmental System Engineering, Sungkyunkwan Univ, Suwon 400- \\ 746, S. Korea ; PH (82) 31-290-7578; FAX (82) 31-290-7570 ; e-mail : cmcic@skku.edu \\ 5 Dept. of Civil, Architectural, and Environmental System Engineering, Sungkyunkwan Univ, Suwon 400- \\ 746, S. Korea ; PH (82) 31-290-7578; FAX (82) 31-290-7570 ; e-mail : yeakim@skku.edu \\ ${ }^{6}$ Doalltech Co., Ltd, DMC Hi-Tech Industry Center 1580, Sangam-Dong, Mapo-Gu, Seoul, Korea 121-835 ; \\ PH (82) 2-555-9779; FAX (82) 2-5555-886 ; e-mail : choi@doalltech.com
}

\begin{abstract}
Recently Building Information Modeling (BIM) has been widely used to manage building information throughout the project life-cycle more effectively and efficiently. Particularly in quantity take-off and estimation, BIM-based process is getting more and more attention, and even BIM-based quantity take-off at the most detailed level has been performed in several building projects in South Korea. The practitioners involved in those projects have pointed out that modeling rough and finish interior of a building occupies a big portion of time in the whole modeling process and the manual modeling for interior is error-prone, which could cause serious result, such as wrong cost estimation and disputes. To resolve the problem, this research proposes an automated modeling method that model a building interior automatically after selecting an interior method by a room or space basis. This research develops a interior modeling method and a system that can model various rough and finish interior components automatically at once after a user select the type of interior for a given space or room. To do so, this research identifies typical interior types of buildings and a house built in South Korea, and develops a mechanism that can support modeling both typical and non-typical types of interior by allowing the flexibility in selecting interior material and components and the order of construction process for the selected items. The automated modeling system for building interior has been preliminarily tested at a typical condominium building project and found that the system could improve dramatically the productivity of BIM-based quantity take-off and estimation process.
\end{abstract}

Keywords: Building Information Modeling (BIM), Three-dimensional CAD model, Quantity Take-off

\section{Introduction}

As Building Information Modeling (BIM) has spread in recent years, many three dimensional Computer Aided Design (CAD) commercial programs used in varied fields such as design, structure, equipment, execution of construction work and estimation have come onto the market. In particular, an application that enables automated quantity take-off from 3D models in the estimation part is examined. BIM based quantity take-off and estimation have been executed by using the application in many projects in South Korea. Also, it is recognized that 3D based estimation applications are better than $2 \mathrm{D}$ based ones in terms of accuracy of quantity and practical use of 3D models. However, it has been discovered by practitioners who worked in the projects that BIM based estimation process needed to be improved while the projects are in progress. One of the factors that leads to that improvement is the task time of the process of modeling the materials 
used in the late stage of building increases when rough/finish interior modeling for the purpose of estimating the precise quantity of materials.

Nowadays, sketches in South Korea construction projects use 2D drawings, therefore, it is necessary to produce 3D models from2D drawings for 3D based quantity take-off. Also, it has been observed that total working time of 3D based estimations take twice longer when using an old estimation system, as 3D modeling takes as much time as estimation does. In addition, it reveals that an effective modeling method is critical since interior finishing modeling takes 90 percent of the total time due to many kinds of materials used. In the case of South Korea, estimation needs a rapid reaction as well as the level of requirements in terms of 3D modeling; otherwise, it becomes a deterrent to induce the country to use 3D modeling.

Hence, this research will recommend a scheme that improves productivity via an automated method that allows solving the problem of increasing total working time when the process of modeling many sorts of materials.

\section{Characteristics of 3D model for quantity take-off}

3D based quantity take-off represents the extraction of quantity data such as length, size, and volume related to quantity from 3D CAD models and connecting the data to the content. Furthermore, this meaning is based on the underlying assumption that materials in the content should be produced for the 3D model or object because there will be no possibility of extracting quantity information without a 3D model.

In the stage of detailed estimation to get the closest quantity to the quantity in real construction projects, exact small quantities for each material are calculated. In order to compute 3D based quantity take-off in the stage of detailed estimation, the modeling process that produces $3 \mathrm{D}$ models for each material constructed is compelling. With the exception of 3D modeling for small supplies such as nails and screws, other major materials have to be modeled according to the content so that the quantity of the content connected with the related materials can be measured.

3D model in the design stage consists of essential elements that determine the room and building exteriors. However, it is very common that this stage does not involve dealing with materials for interiors. Furthermore, using the accomplished model from design stage is impracticable so as to measure the quantity take-off because there are some differences between the 3D model for the quantity take-off and building designs. It is possible to use 3D models from the design stage in the rough estimation stage without any changes. However, it is unworkable to apply it in the phase of calculating detailed small quantities for each material and they needed to be modeled so as to measure the precise quantity take-off.

\section{Limitation of 3D based quantity take-off for construction finishing}

2D based estimation figures out the quantity for each material after referring to the room, measurements coming within the purview of width and length and types of materials for interiors, and then those can be combined detailing the amount of construction expenses. The size coming under the room width and length is standardized according to the interior size of the bony frame, $\mathrm{X}$ and $\mathrm{Y}$ from figure 1 . Also, it is viable to apply the same width to the content and compute the quantity without the classification between materials for the middle phase and the final stage. As materials for the middle phase and the final stage are modeled in the same way as the real construction state from figure 2 , however, the quantity of materials in the very last phase is different depending on the thickness of the materials in the middle stage. Moreover, the finished construction model for 3D based quantity take-off enables not only to estimate the precise amount but also to examine viability of the construction.

For the accurate quantity take-off, materials have to be modeled in the exact state of construction, and, without modeling, it is possible to refine the $3 \mathrm{D}$ model in order to modify wrong quantity measurement. While modeling the materials as well as the bony frame for the purpose of $3 \mathrm{D}$ based quantity take-off for construction finishing, the increase of the task of reforming the model is caused by the increase of the range of modeling. It is feasible that 3D based quantity take-off is admitted in terms of quantity accuracy, on the other hand, it is not efficient and widely applicable in terms of the quickness of the task because the process needs to be formed in 3D model which causes the delay of the task. 


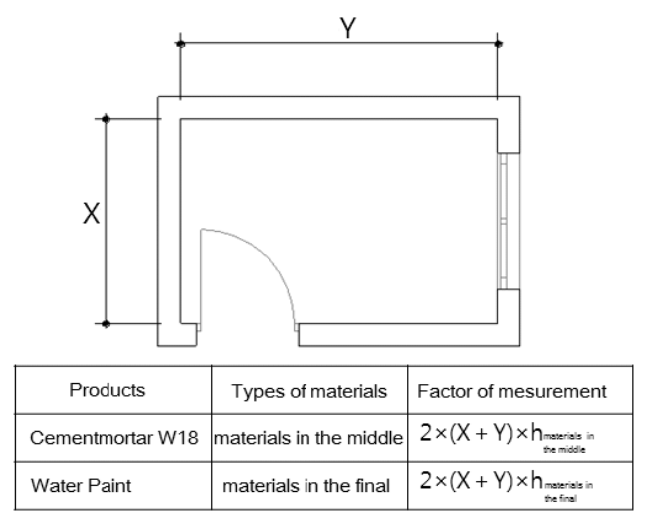

Figure 1.2D based estimation example

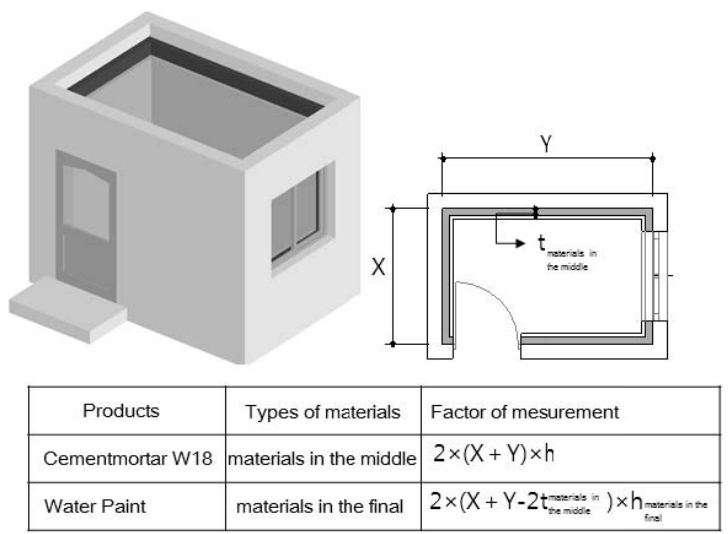

Figure 2. 3D based quantity take-off example

\section{Automated Building Information Modeling System for Building Interiors}

Generally, creating 3D construction modeling consists of elements that determine the shape of rooms. They include historic walls, supports, sleeves and beams which are represented by the building structure and also non-historic walls, blocks and simple frame walls that are used for partitioning. 3D models enable showing similar images of the real construction since it is possible that the model present the quality of the materials. Consequently, it is not viable to take a look at the detailed shape of the materials cutting a section of the 3D model completed at the real stage. This partially results from the fact that it is not common to model every single material at a time in the modeling part, furthermore, it is likely to happen that the single section of the wall including the materials is modeled by an object. It grants to reduce the time of the task to be efficient.

To compute 3D based quantity take-off for construction finishing, it is required to create the 3D model by each type of material with the aim of measuring the quantity as precise as the real state of construction in the detailed part. Also, it is needed to take an action to develop the productivity of the estimation tasks due to the increase of modeling time and the amount of the tasks for the 3D based quantity take-off. Hence, this research formulates the automated building information modeling system to trim down the progress of modeling the materials required and reduce the time of building modeling tasks.

It is very reliable to state that automated building information system enables practitioners to cut the iterated tasks of 3D modeling for the quantity take-off and produce automatically the materials according to the shape of the frame. In other words, it allows practitioners to reduce the amount of the task and the time by using automated system which make internal modeling possible instead of the old method that practitioners repeatedly draw each material followed by the baseline of the frame.(figure 3.) 


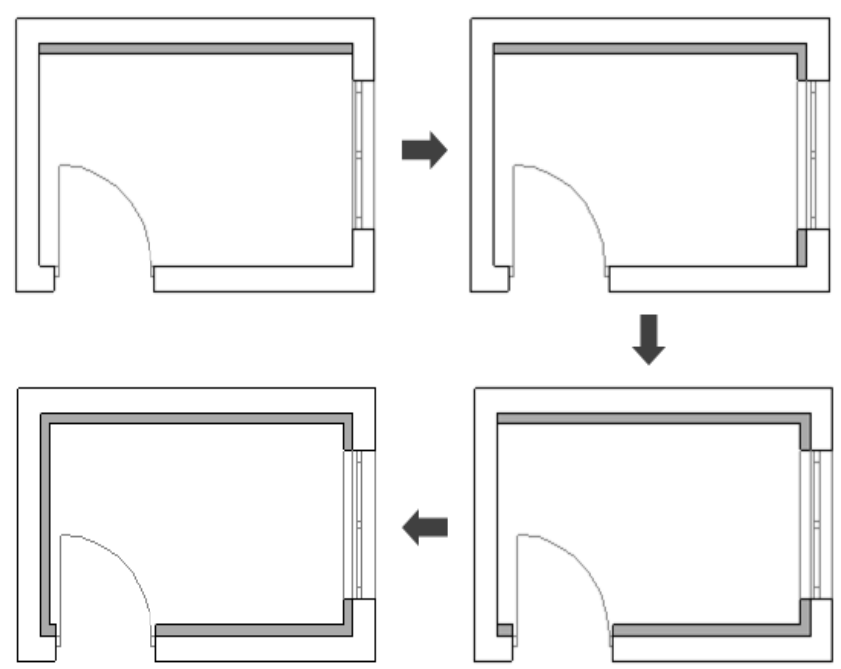

Figure 3. the old method of creating 3D model

\section{Automated Building Information Modeling System Developing and Inspecting}

As indicated, in the old way, practitioners used to draw many materials over and over to create 3D models. Hence, this study has developed an automated building information modeling system to execute drawing tasks internally rather than practitioners drawing manually so that the productivity of the tasks is improved and total time of 3D modeling is reduced.

In the way that practitioners draw manually, the sketches are also decided by their own thoughts; however, it is necessary to interpret the frame area and recognize the baseline for the aim of modeling the materials in the system. Accordingly, the frame of the building up to standard should be set as geometric constraints when modeling materials. The system internally seeks the connection in the frame that users selected and calculate room or coordinates in order to identify the baseline of the finished modeling. Furthermore, it checks the possible spots of the opening and applies to the final stage of the modeling. (Figure 4.)

This system has performed a pilot test which makes it an object of the typical interior modeling of common houses widely used in South Korea so as to examine the result of the system from this study. By testing the system, it has been outlined that the total number of promising modeling is 84 of 211 which is approximately $40 \%$, and about $50 \%$ of the $40 \%$ which is 51 of the materials needed to be improved. Also, 33 materials from the result of the system could be used without any modifying and the time of modeling has been reduced to about $60 \%$ to $70 \%$ compared to manual modeling.

\section{Conclusions}

The research includes the criteria as needed in the stage of detailed estimation for 3D model based quantity take-off and its limitations. To overcome the limits, the finished modeling method that allows to draw many materials at once on the basis of geometric constraint and the Automated Building Information Modeling System based on it have been developed. The system has been trialed to the typical interior modeling of common house which is widely used in South Korea, and, in accordance with the test, higher productivity in terms of 3D based quantity take-off and estimation has been discovered due to the time reduced.

\section{References}

[1] K. Nassar, W. Thabet, Y. Beliveau. (2003). "Building assembly detailing using constraint-based modeling", Automation in Construction, Volume 12, Issue 4, 365-379.

[2] Rafael Sacks, Charles M. Eastman, Ghang Lee. (2004). "Parametric 3D modeling in building construction with examples from precast concrete", Automation in Construction, Volume 13, Issue 3 , 291-312. 
[3] Hilderick A. van der Meiden, Willem F. Bronsvoort. (2007). "Solving topological constraints for declarative families of objects", Computer-Aided Design, Volume 39, Issue 8, 652-662.

[4] Chia-Hui Shih, Bill Anderson. (1997). "A design/constraint model to capture design intent", ACM Symposium on Solid and modeling and applications, 255-264.

[5] Javier Monedero. (2000). "Parametric design: a review and some experiences", Automation in Construction, Volume 9, Issue 4, 369-377.

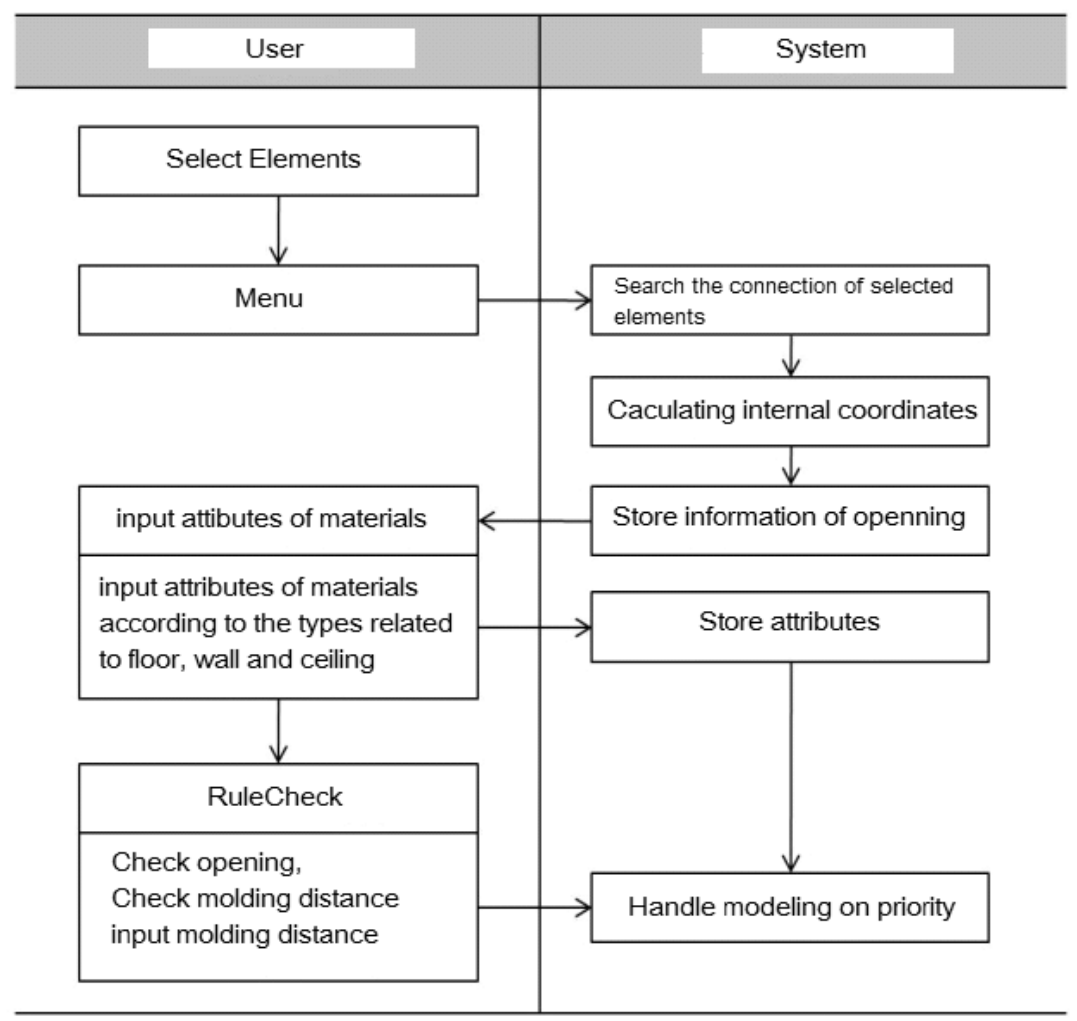

Figure 4. Automated Building Information Modeling System Process for Building Interior

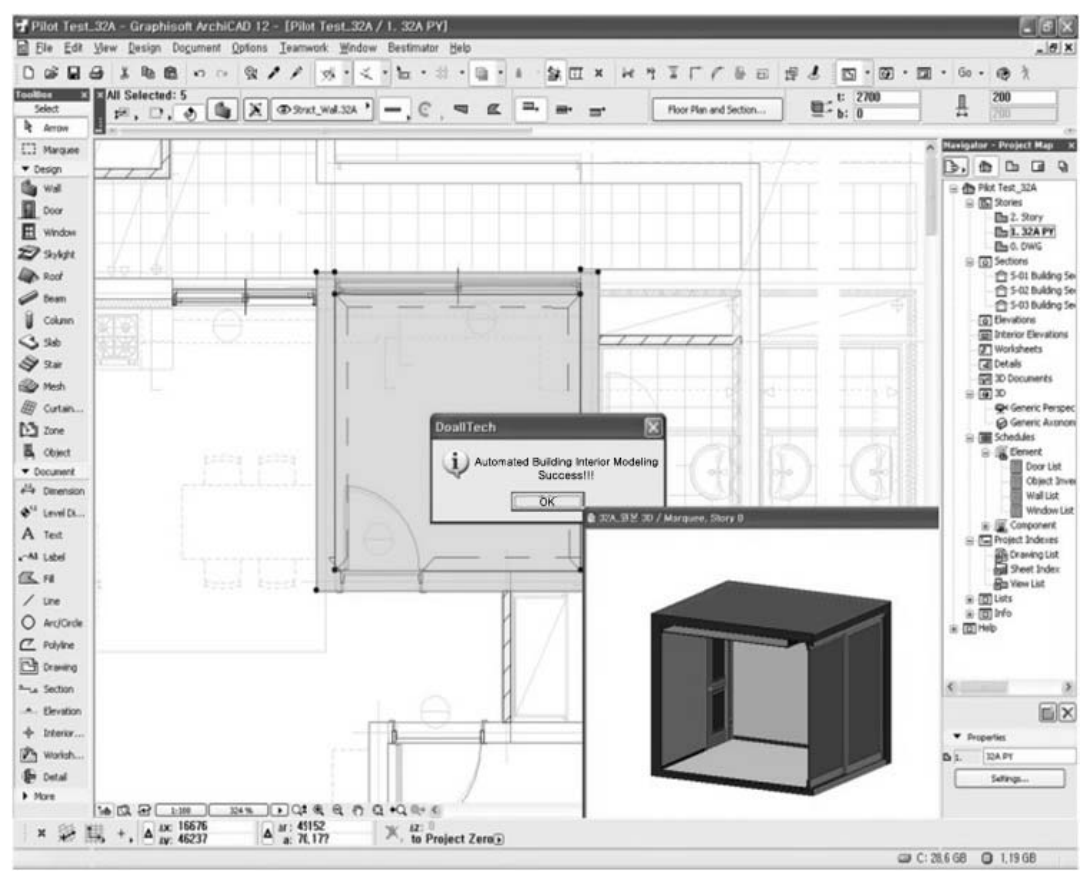

Figure 5. Pilot Test for Automated Building Information Modeling System for Building Interior 